\title{
Changes in plasma levels of perfluoroalkyl substances (PFASs) are related to increase in carotid intima-media thickness over 10 years - a longitudinal study
}

\author{
P. Monica Lind ${ }^{1 *}$ (D, Samira Salihovic ${ }^{2,3}$, Jordan Stubleski ${ }^{3}$, Anna Kärrman ${ }^{3}$ and Lars Lind ${ }^{4}$
}

\begin{abstract}
Background: It has previously been reported that the environmental contaminants perfluoroalkyl substances (PFASs) are linked to atherosclerosis in cross-sectional studies. Since cross-sectional studies could be subject to reverse causation, the purpose of this study was to analyze if the longitudinal changes in PFASs during a 10-year follow-up were related to the change in carotid artery intima-media thickness (IMT, ultrasound) during the same period.

Methods: In the Prospective Investigation of the Vasculature in Uppsala Seniors (PIVUS) study, 1016 individuals were investigated at age 70; 826 of them were reinvestigated at age 75 and 602 at age 80 years. Eight different PFASs were measured in plasma by ultra-performance liquid chromatography-tandem mass spectrometry (HPLC-MS/MS), and IMT was measured at all three time points. Random-effects mixed regression models were used to examine the associations over time.

Results: IMT increased $0.058 \mathrm{~mm}$ during the 10-year period $(p<0.0001)$. Following adjustment for baseline values of PFASs (age 70) and sex, the changes in plasma levels of 6 of the 8 measured PFASs were significantly related to the change in IMT over the 10-year follow-up period in a positive fashion ( $p<0.0062$ using Bonferroni correction for 8 tests). Further adjustment for traditional cardiovascular (CV) risk factors (HDL and LDL cholesterol, smoking, systolic blood pressure, statin use, fasting glucose and serum triglycerides) affected these relationships only marginally.

Conclusion: The change in plasma levels of several PFASs during 10 years was positively related to increase in IMT seen during the same period, giving prospective evidence that PFASs might interfere with the atherosclerotic process.
\end{abstract}

Keywords: Atherosclerosis, Longitudinal, Perfluoroalkyl substances (PFASs), Elderly, Epidemiology, IMT

\section{Background}

Perfluoroalkyl substances (PFASs) belong to a class of organic environmental contaminants that are structurally characterized by a perfluorinated carbon backbone with a terminal functional head group. They are produced in high volumes and used in a variety of applications, ranging from surface-active repellents to fire-fighting foams and cosmetics. Due to their wide use, PFASs have been distributed globally and are detected in blood in almost all individuals in industrialized countries [1-10].

\footnotetext{
* Correspondence: monica.lind@medsci.uu.se

1 Department of Medical Sciences, Occupational and Environmental Medicine, Uppsala University, 75185 Uppsala, Sweden

Full list of author information is available at the end of the article
}

Atherosclerosis is a common disease affecting the majority of individuals in the industrialized countries and could give rise to myocardial infarction and stroke. Several imaging methods to quantify the amount of atherosclerosis are used in research and clinical practice. Of these, ultrasound determinations of the carotid arteries are very common in research; especially the thickness of the intima-media (IMT) complex of the carotid artery wall has been evaluated in many epidemiological studies, and increased IMT has been associated with future myocardial infarction and stroke [11].

One previous publication has reported an association between circulating levels of PFASs and IMT using a cross-sectional approach [12]. Since cross-sectional studies

(c) The Author(s). 2018 Open Access This article is distributed under the terms of the Creative Commons Attribution 4.0 International License (http://creativecommons.org/licenses/by/4.0/), which permits unrestricted use, distribution, and reproduction in any medium, provided you give appropriate credit to the original author(s) and the source, provide a link to the Creative Commons license, and indicate if changes were made. The Creative Commons Public Domain Dedication waiver (http://creativecommons.org/publicdomain/zero/1.0/) applies to the data made available in this article, unless otherwise stated. 
might be subject to reverse causation and confounding, longitudinal studies are preferable. We therefore now present data from the Prospective Investigation of the Vasculature in Uppsala Seniors (PIVUS) study [13], in which the participants were investigated at ages 70, 75 and 80 years. Eight different PFASs and ultrasound-based IMT were measured at the three time points, and the study investigated the hypothesis that PFASs levels were positively related to the change in IMT over the 10-year follow-up.

\section{Methods}

\section{Subjects}

Subjects eligible for inclusion were all individuals aged 70 years living in the municipality of Uppsala, Sweden. The subjects were selected from the municipal register and invited in a randomized order by letter within 2 months of their 70th birthday. Of the 2025 subjects invited, 1016 took part, yielding a participation rate of $50.1 \%$. This baseline investigation was conducted between 2001 and 2004 [13]. All subjects were invited to re-examinations at ages $75(n=826)$ and 80 years $(n=602)$. During the 10-year follow-up, 153 had died.

The study was approved by the Ethics Committee of Uppsala University, and the participants gave informed consent prior to the study.

\section{Basic characteristics and cardiovascular risk factors}

The participants were asked to complete a questionnaire concerning their medical history, smoking habits, and regular medication. All subjects were examined in the morning, having fasted overnight. No medication or smoking was permitted after midnight. Blood pressure was measured using a calibrated mercury sphygmomanometer in the non-cannulated arm to nearest $\mathrm{mmHg}$ after at least $30 \mathrm{~min}$ of rest, and the average of three recordings was used. Lipid variables and fasting blood glucose were determined using standard laboratory techniques [14]. The same methods were applied at all three examinations. Basic cardiovascular risk factors are presented in Table 1 and have been described more in detail in [13].

At baseline, approximately $10 \%$ of the cohort reported a history of coronary heart disease, $4 \%$ reported stroke and $9 \%$ diabetes mellitus. Almost half the cohort reported some cardiovascular medication (45\%), and antihypertensive medication was the most prevalent (32\%). Fifteen percent reported use of statins, while insulin and oral antiglycemic drugs were reported in 2 and 6\%, respectively (for details, see reference [13]).

\section{Carotid artery ultrasound evaluation}

The carotid artery was assessed by external B-mode ultrasound imaging (Acuson XP128 with a $10 \mathrm{MHz}$ linear transducer, Acuson, Mountain View, CA). The images were digitized and imported into the AMS (Artery Measurement
Table 1 Traditional cardiovascular risk factors at the three different investigations in the PIVUS sample

\begin{tabular}{llll}
\hline & Age 70 & Age 75 & Age 80 \\
Variable & Mean (SD) & Mean (SD) & Mean (SD) \\
\hline BMI $\left(\mathrm{kg} / \mathrm{m}^{2}\right)$ & $27.0(4.3)$ & $26.8(4.3)$ & $26.9(4.5)$ \\
Fasting glucose $(\mathrm{mmol} / \mathrm{l})$ & $5.3(1.6)$ & $5.2(1.4)$ & $5.2(1.3)$ \\
Systolic blood pressure $(\mathrm{mmHg})$ & $149(22)$ & $148(19)$ & $146(19)$ \\
LDL cholesterol $(\mathrm{mmol} / \mathrm{l})$ & $3.38(0.88)$ & $3.37(0.94)$ & $3.2(0.9)$ \\
HDL cholesterol $(\mathrm{mmol} / \mathrm{l})$ & $1.51(0.43)$ & $1.49(0.46)$ & $1.38(0.39)$ \\
Smokers (\%) & 11 & 6 & 3 \\
Statin use (\%) & 15 & 25 & 30 \\
\hline
\end{tabular}

$B M I$ body mass index, $S D$ standard deviation, $H D L$ high-density lipoprotein, LDL low-density lipoprotein

Software) automated software for dedicated analysis of IMT in the far wall in the common carotid artery (CCA) 1-2 $\mathrm{cm}$ proximal to the bulb. A 10-mm segment with good image quality was chosen for analysis. The program automatically discerns the borders of the intima-media thickness of the far wall and the inner diameter of the vessel and computes intima-media thickness and the diameter from roughly 100 discrete measurements through the 10 -mm-long segment. The value given for IMT is the mean value from both sides. The measurements of intima-media thickness were repeated in 30 random subjects yielding a coefficient of variation of carotid artery intima-media thickness of $7.2 \%$ [15].

\section{Methods for the chemical analysis of the PFASs}

The analytical procedure used for all plasma samples $(150 \mu \mathrm{L})$ in the present study involves rapid protein and phospholipid removal using Ostro 96-well plates (Waters, Milford, MA). Following extraction, the samples were transferred to vials and analyzed using large volume injection (LVI) automated column-switching ultra-performance liquid chromatography-tandem mass spectrometry (HPLC-MS/MS, Waters, Milford, MA). In total, the method enabled the assessment of 14 PFASs. For this study, however, only PFASs with detection rates $>75 \%$ were used in subsequent statistical analyses: perfluoroheptanoic acid (PFHpA), perfluorononanoic acid (PFNA), perfluorodecanoic acid (PFDA), perfluoroundecanoic acid (PFUnDA), perfluorohexane sulfonic acid (PFHxS), linear isomer of perfluorooctane sulfonic acid (PFOS), perfluorooctane sulfonic acid (PFOA) and perfluorooctane sulfonamide (PFOSA). Detailed information about the analytical procedure and method performance is presented in [16].

\section{Statistical analyses}

All eight PFASs were skewed towards high levels but were normally distributed following ln-transformation. The same trend was also observed for fasting blood glucose 
and serum triglycerides. Therefore, the ln-transformed values were used in the statistical models.

First, the change in IMT over 10 years (three measurements) were evaluated by mixed random effect models with IMT as dependent variable, and time as the independent variable and sex as confounder (age same in all subjects).

Thereafter, the relationships between changes over 10 years in the levels of the eight PFASs and the changes over 10 years in IMT were examined. Also for this purpose mixed random effect models were used, but here each PFAS was used as an independent variable. The independent variable was split into a between-individual component, which is the first observation for the individual, and a within-individual component, which is the difference between the measurements at future time points and the first measurement. Thus, the between-individual component is related to the mean of the three measurements of IMT, while the between-individual component, as a single term, relates the change in each PFAS to the change in IMT. The theory and assumptions behind this model as well as the detailed formula is given at page 420 in Applied longitudinal analysis by Fitzmaurice, G.M. et al. [17]. The general formula is; $Y_{i j}=Z_{i} b e t a_{0}-X_{i 1}$ beta $_{C}+\left(X_{i j}-X_{i 1}\right) b_{e t a}+e_{i j}$, where $Y$ is IMT, $X$ is the PFAS, $i$ is the individual, $j$ the time, beta $_{C}$ is the coefficient for the first observation and beta $a_{L}$ is the coefficient for change over time. Confounders and the intercept are given as $Z_{i} b^{b} a_{0}$. The general formula we used in STATA for the first set of models was; mixed IMT PFASchange PFAS1 sex || id:, where 1 denotes the first observation at age 70 . We used only random intercept, since inclusion of also a random slope made the models not to converge in most cases. The random part of the model is the identity of the subjects and the rest of the model are fixed effects. We used the default covariance structure of the command "mixed", which is independent, which is appropriate for mixed models with a random intercept.

The first set of models (one for each PFAS) were adjusted for sex and the baseline values for the PFASs. The second set of models were also adjusted for traditional cardiovascular risk factors (HDL and LDL cholesterol, smoking, systolic blood pressure, BMI, fasting glucose and serum triglycerides) and statin use.

An interaction term between sex and the PFASs was included in the first set of models to determine any sex interaction regarding IMT.

STATA14 (Stata Inc., College Station, TX) were used for calculations. Since we investigated 8 PFASs, we applied $p<0.00625(0.05 / 8)$ as the level of significance.

\section{Results}

Distributions of traditional $\mathrm{CV}$ risk factors are summarized in Table 1. Median IMT at age 70 was $0.89 \mathrm{~mm}$ (SD 0.16). An increase in IMT by $0.058 \mathrm{~mm}$ (SD $0.043, p<0.0001$ ) was observed during the 10-year follow-up. This is well in line with a meta-analysis of 22 studies in which the annual change in IMT ranged from 0.001 to $0.030 \mathrm{~mm}$ [18].

The baseline levels of the PFAS are given in Table 2. The results of the longitudinal changes in the eight PFASs have been given in detail in a previous publication [19]. In summary, over the 10- year follow-up, PFHxS, PFUnDA, PFDA, and PFNA levels increased (ranging from 7 to 34\%), while PFOA, PFHpA, PFOS, and PFOSA levels were reduced (ranging from $-75 \%$ to $-27 \%$ ) in a significant fashion.

A Spearman rank correlation matrix for the changes from age 70 to age 80 regarding the 8 PFAS are given in Table 3.

The changes in plasma levels of all eight PFASs over the 10 years were related to the change in IMT during the same period (when analyzed one by one), adjusting for baseline levels of the PFASs and sex, when $p<0.05$ was used. Using a Bonferroni-corrected $p$-value for eight tests $(p<0.00625)$, six of the eight PFASs were still significant, as can be seen in Table 4 .

Further adjustments for traditional cardiovascular risk factors changed these relationships only marginally. In these fully adjusted models, baseline PFASs levels were not significantly related to the change in IMT ( $p>0.05$ for all).

As could be seen in Table 4 in detail, a change of one interquartile range (IQR) in the change of PFAS levels (being $0.05 \mathrm{ng} / \mathrm{ml}$ for PFHpA, 5.7 for PFHxS, 5.6 of PFOS, 1.3 for PFOA, 0.40 for PFNA, 0.15 for PFDA, 0.09 for PFOSA and $0.19 \mathrm{ng} / \mathrm{mlfor}$ PFUnDA) over the 10 -year period corresponded to a change in IMT by $0.0056-0.016 \mathrm{~mm}$.

No significant interactions were found between sex and the change in the eight PFASs regarding the change in IMT.

The relationships between the changes in plasma levels of the PFASs over the 10 years were related to the change in IMT during the same period. Fig. 1 shows the associations between three different PFASs (PFHpA, PFOA, and PFUnDA) whose chemical structure differs only in the number of carbon-fluorine bonds.

Table 2 Median and interquartile range for the eight studied PFAS at the baseline investigation (at age 70) in $\mathrm{ng} / \mathrm{ml}$

\begin{tabular}{ll}
\hline PFHPA & $0.05(0.03,0.09)$ \\
PFHXS & $2.08(1.6,3.42)$ \\
PFOS & $13.23(9.95,17.77)$ \\
PFOA & $3.3(2.52,4.39)$ \\
PFNA & $0.70(0.52,0.97)$ \\
PFDA & $0.31(0.24,0.40)$ \\
PFOSA & $0.11(0.07,0.17)$ \\
PFUnDA & $0.28(0.22,0.37)$ \\
\hline
\end{tabular}

PFASs: PFHpA perfluoroheptanoic acid, $P F H x S$ perfluorohexane sulfonic acid, PFOS perfluorooctane sulfonic acid, PFOA perfluorooctane sulfonic acid, PFNA perfluorononanoic acid, PFDA perfluorodecanoic acid, PFOSA perfluorooctane sulfonamide, PFUnDA perfluoroundecanoic acid 
Table 3 Spearman rank correlation for relationships between the changes from age 70 to age 80 for the different PFAS studied

\begin{tabular}{llllllll}
\hline & PFHpA & PFHxS & PFOS & PFOA & PFNA & PFDA & PFOSA \\
\hline PFHXS & 0.15 & & & & & & \\
PFOS & 0.20 & 0.20 & & & & & \\
PFOA & 0.35 & 0.35 & 0.50 & & & & \\
PFNA & 0.19 & 0.22 & 0.35 & 0.46 & & & \\
PFDA & 0.18 & 0.20 & 0.38 & 0.41 & 0.75 & & \\
PFOSA & 0.20 & 0.04 & 0.36 & 0.28 & 0.12 & 0.13 & \\
PFUnDA & 0.09 & 0.21 & 0.31 & 0.31 & 0.57 & 0.76 & 0.09
\end{tabular}

PFASs: PFHpA, perfluoroheptanoic acid, $P F H \times S$ perfluorohexane sulfonic acid, PFOS perfluorooctane sulfonic acid, PFOA perfluorooctane sulfonic acid, PFNA perfluorononanoic acid, PFDA perfluorodecanoic acid, PFOSA perfluorooctane sulfonamide, PFUnDA perfluoroundecanoic acid

\section{Discussion}

The present longitudinal study showed that the changes in plasma levels of several PFASs were related to the change in IMT during a 10-year follow-up period in a sample of elderly subjects, providing further evidence that PFASs could interfere with the atherosclerotic process. Furthermore, adjustment for the traditional cardiovascular risk factors changed these results only marginally, suggesting that a possible effect of PFASs on IMT is not mediated by the traditional risk factors. In addition, this study adds to previously published studies showing that other environmental contaminants, like the PCBs [20], some phthalates [21], as well as some metals [22], are also linked to atherosclerosis.

In a previous population-based cross-sectional study of Taiwanese adolescents and young adults a positive relationship between PFOS and IMT was found, while the study reported a negative relationship between PFUnDA and IMT [12]. This group also showed that this association was more prominent in individuals with elevated levels of circulating endothelial and platelet microparticles [23]. In the present study, we found that both PFOS and PFUnDA, and some other PFASs, were related to IMT. The reason for this discrepancy between the studies is not known, but might be due to the fact that the studies were conducted in very different age-groups and in countries with different exposures of the PFASs.

Since cross-sectional studies might be subject to reverse causation and confounding, longitudinal studies are preferable. Therefore, the present longitudinal study provides a higher level of evidence that PFASs could interfere with atherosclerosis formation, although only randomized interventions could provide the final evidence in this respect. It is interesting to note that in a cross-sectional analysis of the same study at the baseline investigation at age 70 we did not find any significant relationship between PFASs and IMT [24]. This

Table 4 Relationships between changes in plasma levels of eight PFAS over 10 years and change in IMT over 10 years. Both the PFASs and IMT were measured at age 70, 75, and 80 years. All models were adjusted for sex. In the multiple adjusted models, additional adjustment was performed for HDL and LDL cholesterol, smoking, systolic blood pressure, statin use, BMI, fasting glucose and serum triglycerides at the three time points

\begin{tabular}{|c|c|c|c|c|c|c|}
\hline & Beta & SE & z & $p$-value & [95\% Conf. Interval] & Change in IMT for an IQR change in the PFAS \\
\hline PFHpA sex-adjusted & 0.010 & 0.003 & 3.05 & 0.0023 & $0.004-0.017$ & \\
\hline PFHpA multiple-adjusted & 0.011 & 0.003 & 3.13 & 0.0017 & $0.004-0.017$ & 0.016 \\
\hline PFHxS sex-adjusted & 0.014 & 0.005 & 2.77 & 0.0057 & $0.004-0.024$ & \\
\hline PFHxS multiple-adjusted & 0.015 & 0.005 & 2.99 & 0.0028 & $0.005-0.025$ & 0.012 \\
\hline PFOS sex-adjusted & 0.011 & 0.005 & 2.35 & 0.019 & $0.002-0.020$ & \\
\hline PFOS multiple-adjusted & 0.011 & 0.005 & 2.35 & 0.019 & $0.002-0.020$ & 0.0056 \\
\hline PFOA sex-adjusted & 0.021 & 0.006 & 3.29 & 0.0010 & $0.008-0.033$ & \\
\hline PFOA multiple-adjusted & 0.021 & 0.006 & 3.23 & 0.0012 & $0.008-0.033$ & 0.0088 \\
\hline PFNA sex-adjusted & 0.016 & 0.006 & 2.68 & 0.0074 & $0.004-0.028$ & \\
\hline PFNA multiple-adjusted & 0.017 & 0.006 & 2.69 & 0.0072 & $0.005-0.028$ & 0.0077 \\
\hline PFDA sex-adjusted & 0.020 & 0.007 & 2.93 & 0.0034 & $0.007-0.033$ & \\
\hline PFDA multiple-adjusted & 0.020 & 0.007 & 2.89 & 0.0038 & $0.006-0.033$ & 0.0089 \\
\hline PFOSA sex-adjusted & 0.011 & 0.004 & 2.84 & 0.0045 & $0.004-0.020$ & \\
\hline PFOSA multiple-adjusted & 0.020 & 0.004 & 3.06 & 0.0022 & $0.005-0.021$ & 0.016 \\
\hline PFUnDA sex-adjusted & 0.022 & 0.007 & 3.10 & 0.0020 & $0.008-0.036$ & \\
\hline PFUnDA multiple-adjusted & 0.023 & 0.007 & 3.26 & 0.0011 & $0.009-0.037$ & 0.011 \\
\hline
\end{tabular}

Ln-transformed values for the PFASs are used in the models. The column at the far right shows the change in IMT (mm) that corresponds to a one interquartile range (IQR) change in the PFAS (just given to the multiple adjusted models

PFASs: PFHpA perfluoroheptanoic acid, PFHXS perfluorohexane sulfonic acid, PFOS perfluorooctane sulfonic acid, PFOA perfluorooctane sulfonic acid, PFNA perfluorononanoic acid, PFDA perfluorodecanoic acid, PFOSA perfluorooctane sulfonamide, PFUnDA perfluoroundecanoic acid 


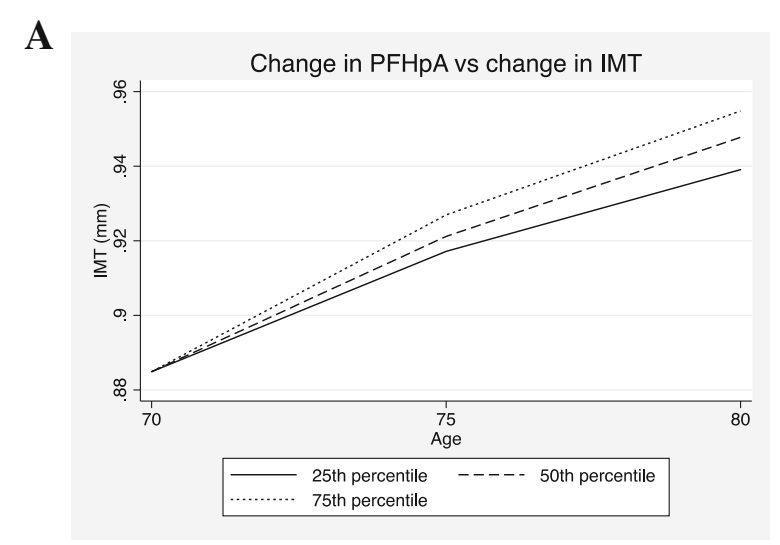

B

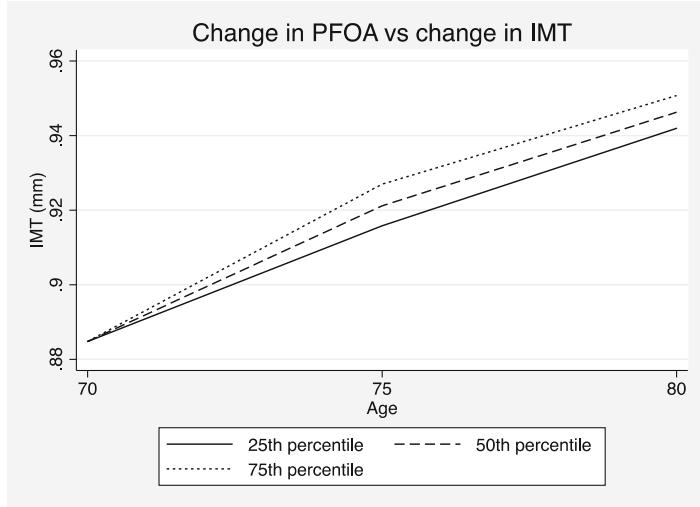

C

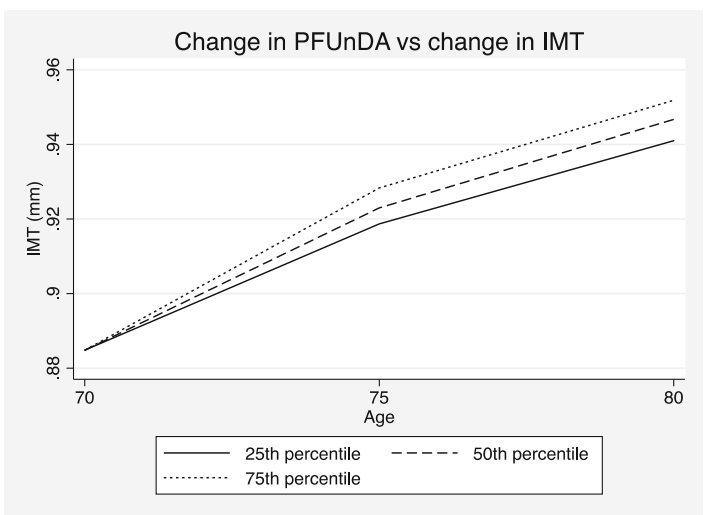

Fig. 1 Relationships between the changes in PFHpA (a, upper panel), PFOA (b, middle panel) and PFUnDA (c, lower panel) and the change in carotid intima-media thickness (IMT) from age 70 to age 80 years. The change in the three perfluoroalkyl substances (PFASs) is given for three levels of change, the 25th percentile, the 50th percentile and the 75 th percentile. The baseline value (age 70 years) is set the same for all levels of change in PFASs to improve graphic interpretation

exemplifies that the longitudinal approach is far superior to the cross-sectional approach, since it also increases the statistical power, as the within-individual variation is usually lower than the between-individual variation.
As a sensitivity analysis, we performed an analysis on the relationships between the changes in PFASs and change in IMT only in the 579 subjects attending all three examinations and the effect estimates, the betas, were almost identical although the confidence intervals were wider due to the small number of observations. Thus, loss of follow-up cannot explain the relationships found in the present study.

The mechanism behind the suggestive effect of PFASs on carotid atherosclerosis is not known. PFASs are known ligands of the nuclear receptors PPAR alpha and gamma [25], and could thereby influence lipids, adipose tissue, and glucose homeostasis. It has previously been shown that pharmaceutical PPAR-gamma ligands, such as thiazolidinediones, could influence IMT [26], so an effect of other PPAR ligands, like PFASs, on IMT is not surprising. It has also been repeatedly shown that PFASs influence cholesterol metabolism [27]. Furthermore, an immune reaction with subsequent inflammation is considered a cornerstone in atherosclerosis development [28]. From that perspective, it is interesting that exposure to PFASs has been associated with a number of perturbations in the immune system in experimental studies [29-31]. Another recently highlighted mechanism of PFASs is that PFASs might influence circulating microparticles, being markers of endothelial dysfunction constituting an early step in atherosclerosis formation [23]. Thus, since many mechanisms could potentially link PFASs exposure to atherosclerosis, we hope that the previous and present findings of a link between PFASs and atherosclerosis in humans will inspire future experimental work to gain further mechanistic insights.

Correlating the changes in the PFASs to each other, we can see that most of those, especially the long-chained PFDA, PFNA and PFUnDA are highly correlated (Table 3). Thus, it is not easy in this setting to establish if the change in a specific PFASs is related to the change in IMT or if it is a class effect. Also in this case, experimental studies are needed.

The fact that correlations generally were seen between the changes in the eight PFASs might explain the fact that a positive association was seen between the change in most of the PFASs studied and the change in IMT despite the fact that some of the PFASs declined over the 10 year period and some increased.

IMT has previously been associated with future myocardial infarction and stroke [11]. In a recent publication, also the predictive power of the change in IMT has been evaluated in a meta-analysis of several studies based on individual data [18] and it was found that baseline IMT was related to cardiovascular events, but not the change in IMT. In that study the follow-up period was shorter (median 4 years) than in the present study (10 years). However, the clinical implications of the 
found relationships between the change in PFASs and the change in IMT are not obvious.

In the models where both the baseline PFASs levels and the change in PFAS were included, only the change in PFASs were significantly related to the change in IMT. This might indicate that changes in PFASs levels over a fairly short time-frame could be of importance for IMT, but it might also be due to the fact that from a statistical view the within-subject variation (change in PFASs) is by far a more powerful determinant of a change (in IMT) than the between-subject variation (baseline PFASs).

The mean IMT increase over the 10 years was $0.06 \mathrm{~mm}$. Eighty-five percent of this increase occurred during the first 10 years. As given in detail in ref. 17, a general increase in the PFASs were seen during these first 5 years. However, during the second 5 -year period, the levels of the PFASs generally went down, which might be an explanation to why the increase in IMT over the last 5 years was very small. During this period, we also noted a decline in IMT in a number of subjects.

The number of carbon-fluorine bonds constituting the different PFASs analogues is a major determinant of the half-life of the PFASs in humans. Overall, experimental studies have reported that both persistence and toxicity tend to increase with increasing numbers of carbon-fluorine bonds. In the present study, eight PFASs consisting of six to eleven carbons $\left(\mathrm{C}_{6}-\mathrm{C}_{11}\right)$ were evaluated, and six were significantly related to IMT in a similar fashion (see Fig. 1).

We have previously shown that some PFASs levels were related to plaque prevalence. However, we think the change in plaque prevalence over a limited time period is not a good measure of the change in the amount atherosclerosis. For that purpose we need a more graded measure of plaque, like the plaque area, which unfortunately is not available over time in the PIVUS study. Furthermore, in the subjects with 2 carotid plaques (about $1 / 3$ of the population) any progression cannot be evaluated, and this part of the population is probably the most important to study.

The strength of the present study is the repeated measurements of PFASs, traditional cardiovascular risk factors, and IMT at three time points over 10 years in a fairly large sample. One limitation is that the sample consists of elderly Swedes, which limits the generalizability.

\section{Conclusions}

In conclusion, the changes in plasma levels of six out of the eight PFASs evaluated during 10 years were related to the increase in IMT seen during the same period, representing prospective evidence that PFASs might interfere with the atherosclerotic process.

\section{Abbreviations}

AMS: Artery Measurement Software; CCA: Common carotid artery;

CV: Cardiovascular; FORMAS: Swedish Research Council for Environment, Agricultural Sciences and Spatial Planning; HDL: High-density lipoprotein; HPLC-MS/MS: Ultra-performance liquid chromatography-tandem mass spectrometry; IMT: Intima-media thickness; LDL: Low-density lipoprotein; LVI: Large volume injection; PFASs: Perfluoroalkyl substances;

PFDA: Perfluorodecanoic acid; PFHpA: Perfluoroheptanoic acid; PFHXS: Perfluorohexane sulfonic acid; PFNA: Perfluorononanoic acid; PFOA: Perfluorooctane sulfonic acid; PFOS: Linear isomer of perfluorooctane sulfonic acid; PFOSA: Perfluorooctane sulfonamide; PFUnDA: Perfluoroundecanoic acid; PIVUS: Prospective Investigation of the Vasculature in Uppsala Seniors; SD: Standard deviation

\section{Acknowledgments}

We gratefully acknowledge the contributions from participants, researchers and others engaged in the PIVUS study. Erik Lampa is acknowledged for his valuable contribution of a script for the figures.

\section{Funding}

This study was supported by the Swedish Research Council for Environment, Agricultural Sciences and Spatial Planning (FORMAS, grant number 2007-2047).

\section{Availability of data and materials}

The data used for this study are not publicly available because consent was not obtained from participants to do so.

\section{Authors' contributions}

ML conceived the project, wrote the initial draft of the manuscript and revised the manuscript. JS, SS and AK performed the chemical analysis data and contributed to critical revision of the manuscript for important intellectual content. LL is principal investigator of PIVUS, performed the data analysis and contributed to critical revision of the manuscript for important intellectual content. Also, LL had full access to all the data in the study and takes responsibility for the integrity of the data and the accuracy of the data analysis. All authors read and approved the final manuscript.

\section{Ethics approval and consent to participate}

The study was approved by the Ethics Committee of Uppsala University, and the participants gave informed consent prior to the study.

\section{Consent for publication}

Not applicable

\section{Competing interests}

The authors declare that they have no competing interests.

\section{Publisher's Note}

Springer Nature remains neutral with regard to jurisdictional claims in published maps and institutional affiliations.

\section{Author details}

${ }^{1}$ Department of Medical Sciences, Occupational and Environmental Medicine, Uppsala University, 75185 Uppsala, Sweden. ${ }^{2}$ Department of Medical

Sciences, Molecular Epidemiology and Science for Life Laboratory, Uppsala University, Uppsala, Sweden. ${ }^{3}$ MTM Research Center, School of Science and Technology, Örebro University, Örebro, Sweden. ${ }^{4}$ Department of Medical Sciences, Cardiovascular Epidemiology, Uppsala University, Uppsala, Sweden.

Received: 16 March 2018 Accepted: 15 June 2018

Published online: 03 July 2018

References

1. Salihovic S, Karrman A, Lind L, Lind PM, Lindstrom G, van Bavel B. Perfluoroalkyl substances (PFAS) including structural PFOS isomers in plasma from elderly men and women from Sweden: results from the Prospective Investigation of the Vasculature in Uppsala Seniors (PIVUS). Environ Int. 2015;82:21-7.

2. Fromme H, Tittlemier SA, Volkel W, Wilhelm M, Twardella D. Perfluorinated compounds-exposure assessment for the general population in Western countries. Int J Hyg Environ Health. 2009;212(3):239-70. 
3. Glynn A, Berger U, Bignert A, Ullah S, Aune M, Lignell S, Darnerud PO. Perfluorinated alkyl acids in blood serum from primiparous women in Sweden: serial sampling during pregnancy and nursing, and temporal trends 1996-2010. Environ Sci Technol. 2012;46(16):9071-9.

4. Kato K, Wong LY, Jia LT, Kuklenyik Z, Calafat AM. Trends in exposure to polyfluoroalkyl chemicals in the US population: 1999-2008. Environ Sci Technol. 2011;45(19):8037-45

5. Harris MH, Rifas-Shiman SL, Calafat AM, Ye X, Mora AM, Webster TF, Oken E, Sagiv SK. Predictors of per- and Polyfluoroalkyl Substance (PFAS) plasma concentrations in 6-10 year old American children. Environ Sci Technol. 2017;51(9):5193-204

6. Fromme H, Wockner M, Roscher E, Volkel W. ADONA and perfluoroalkylated substances in plasma samples of German blood donors living in South Germany. Int J Hyg Environ Health. 2017;220(2 Pt B):455-60.

7. Eriksson U, Mueller JF, Toms LL, Hobson P, Karrman A. Temporal trends of PFSAs, PFCAs and selected precursors in Australian serum from 2002 to 2013. Environ Pollut. 2017;220(Pt A):168-77.

8. Poothong S, Thomsen C, Padilla-Sanchez JA, Papadopoulou E, Haug LS. Distribution of novel and well-known poly- and Perfluoroalkyl Substances (PFASs) in human serum, plasma, and whole blood. Environ Sci Technol. 2017:51:13388-96.

9. Sochorova L, Hanzlikova L, Cerna M, Drgacova A, Fialova A, Svarcova A, Gramblicka T, Pulkrabova J. Perfluorinated alkylated substances and brominated flame retardants in serum of the Czech adult population. Int J Hyg Environ Health. 2017;220(2 Pt A):235-43.

10. Lee $\mathrm{JH}$, Lee $\mathrm{CK}$, Suh $\mathrm{CH}$, Kang HS, Hong CP, Choi SN. Serum concentrations of per- and poly-fluoroalkyl substances and factors associated with exposure in the general adult population in South Korea. Int J Hyg Environ Health. 2017;220(6):1046-54.

11. O'Leary DH, Polak JF, Kronmal RA, Manolio TA, Burke GL, Wolfson SK, Grp CHSCR. Carotid-artery intima and media thickness as a risk factor for myocardial infarction and stroke in older adults. N Engl J Med. 1999;340(1):14-22.

12. Lin CY, Lin LY, Wen TW, Lien GW, Chien KL, Hsu SHJ, Liao CC, Sung FC, Chen PC, Su TC. Association between levels of serum perfluorooctane sulfate and carotid artery intima-media thickness in adolescents and young adults. Int J Cardiol. 2013;168(4):3309-16.

13. Lind L, Fors N, Hall J, Marttala K, Stenborg A. A comparison of three different methods to evaluate endothelium-dependent vasodilation in the elderly the Prospective Investigation of the Vasculature in Uppsala Seniors (PIVUS) study. Arterioscler Thromb Vasc Biol. 2005;25(11):2368-75.

14. Carlsson L, Lind L, Larsson A. Reference values for 27 clinical chemistry tests in 70-year-old males and females. Gerontology. 2010;56(3):259-65.

15. Hulthe J, Wikstrand J, Emanuelsson H, Wiklund O, deFeyter PJ, Wendelhag I. Atherosclerotic changes in the carotid artery bulb as measured by B-mode ultrasound are associated with the extent of coronary atherosclerosis. Stroke. 1997;28(6):1189-94.

16. Salihovic S, Karrman A, Lindstrom G, Lind PM, Lind L, van Bavel B. A rapid method for the determination of perfluoroalkyl substances including structural isomers of perfluorooctane sulfonic acid in human serum using 96-well plates and column-switching ultra-high performance liquid chromatography tandem mass spectrometry. J Chromatogr A. 2013;1305:164-70.

17. Fitzmaurice GM, Laird NM, Ware JH. Applied longitudinal analysis. 2nd ed. Hoboken: Wiley; 2011.

18. Lorenz MW, Polak JF, Kavousi M, Mathiesen EB, Volzke H, Tuomainen TP, Sander D, Plichart M, Catapano AL, Robertson CM, et al. Carotid intimamedia thickness progression to predict cardiovascular events in the general population (the PROG-IMT collaborative project): a meta-analysis of individual participant data. Lancet. 2012;379(9831):2053-62.

19. Stubleski J, Salihovic S, Lind L, Lind PM, van Bavel B, Karrman A. Changes in serum levels of perfluoroalkyl substances during a 10-year follow-up period in a large population-based cohort. Environ Int. 2016;95:86-92.

20. Lind PM, van Bavel B, Salihovic S, Lind L. Circulating levels of persistent organic pollutants (POPS) and carotid atherosclerosis in the elderly. Environ Health Perspect. 2012;120(1):38-43.

21. Lind PM, Lind L. Circulating levels of bisphenol $A$ and phthalates are related to carotid atherosclerosis in the elderly. Atherosclerosis. 2011;218(1):207-13.

22. Lind PM, Olsen L, Lind L. Circulating levels of metals are related to carotid atherosclerosis in elderly. Sci Total Environ. 2012;416:80-8.

23. Lin CY, Chen PC, Lo SC, Torng PL, Sung FC, Su TC. The association of carotid intima-media thickness with serum level of perfluorinated chemicals and endothelium-platelet microparticles in adolescents and young adults. Environ Int. 2016:94:292-9.

24. Lind PM, Salihovic S, van Bavel B, Lind L. Circulating levels of perfluoroalkyl substances (PFASs) and carotid artery atherosclerosis. Environ Res. 2017;152:157-64

25. Lau C, Anitole K, Hodes C, Lai D, Pfahles-Hutchens A, Seed J. Perfluoroalkyl acids: a review of monitoring and toxicological findings. Toxicol Sci. 2007; 99(2):366-94.

26. Saremi A, Schwenke DC, Buchanan TA, Hodis HN, Mack WJ, Banerji M, Bray GA, Clement SC, Henry RR, Kitabchi AE, et al. Pioglitazone slows progression of atherosclerosis in prediabetes independent of changes in cardiovascular risk factors. Arterioscler Thromb Vasc Biol. 2013;33(2):393-9.

27. Nelson JW, Hatch EE, Webster TF. Exposure to polyfluoroalkyl chemicals and cholesterol, body weight, and insulin resistance in the general U.S. population. Environ Health Perspect. 2010;118(2):197-202.

28. Libby $P$, Hansson GK. Inflammation and immunity in diseases of the arterial tree: players and layers. Circ Res. 2015;116(2):307-11.

29. Ryu MH, Jha A, Ojo OO, Mahood TH, Basu S, Detillieux KA, Nikoobakht N, Wong CS, Loewen M, Becker AB, et al. Chronic exposure to perfluorinated compounds: impact on airway hyperresponsiveness and inflammation. Am J Physiol Lung Cell Mol Physiol. 2014;307(10):L765-74.

30. DeWitt JC, Williams WC, Creech NJ, Luebke RW. Suppression of antigen-specific antibody responses in mice exposed to perfluorooctanoic acid: role of PPARalpha and T- and B-cell targeting. J Immunotoxicol. 2016;13(1):38-45.

31. Zhang H, Fang W, Wang D, Gao N, Ding Y, Chen C. The role of interleukin family in perfluorooctanoic acid (PFOA)-induced immunotoxicity. J Hazard Mater. 2014;280:552-60

\section{Ready to submit your research? Choose BMC and benefit from:}

- fast, convenient online submission

- thorough peer review by experienced researchers in your field

- rapid publication on acceptance

- support for research data, including large and complex data types

- gold Open Access which fosters wider collaboration and increased citations

- maximum visibility for your research: over $100 \mathrm{M}$ website views per year

At BMC, research is always in progress.

Learn more biomedcentral.com/submissions 